\title{
Urticaceae endémicas del Perú
}

\section{Blanca León ${ }^{1,2}$}

${ }^{1}$ Museo de Historia Natural, Av. Arenales 1256, Aptdo. 14-0434, Lima 14, Perú

2 Plant Resources Center, University of Texas at Austin, Austin TX 78712 EE.UU.

blanca.leon@mail.utexas.edu

\section{Resumen}

La familia Urticaceae es reconocida en el Perú por presentar 10 géneros y 87 especies (Brako \& Zarucchi, 1993; Ulloa Ulloa et al., 2004), entre hierbas, arbustos y árboles. En este trabajo reconocemos como endemismos a 24 especies y dos taxones subespecíficos, en seis géneros. Pilea es el género más rico en especies endémicas. Los taxones endémicos han sido encontrados principalmente en las regiones Bosques Muy Húmedos Montanos y Mesoandina, entre los 1600 y 3550 m de altitud. Se aplicaron las categorías y criterios de la UICN a seis especies. Dos especies han sido registradas dentro del Sistema Nacional de Áreas Naturales Protegidas por el Estado.

Palabras claves: Urticaceae, Pilea, Perú, endemismo, plantas endémicas.

\section{Abstract}

The Urticaceae are represented in Peru by ten genera and 87 species (Brako \& Zarucchi, 1993; Ulloa Ulloa et al., 2004), between herbs, shrubs and trees. Here we recognize 24 species and two infra-specific taxa as endemics, representing six genera. Pilea is the genus with more endemic species. Endemic taxa are found mainly in Very Humid Montane Forests and Mesoandean regions, between 1600 and 3550 m elevation. We applied IUCN categories and criteria to six species. Two endemic Urticaceae have been recorded within Peru's protected areas system.

Keywords: Urticaceae, Pilea, Peru, endemism, endemic plants.

\section{Boehmeria bullata Kunth subsp. notobullata Friis \&} Wilmot-Dear

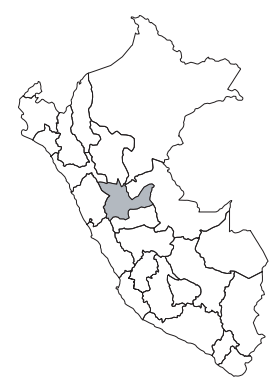

Publicación: Opera Bot. 129: 34-35, f. 11 A-K. 1996.

Colección tipo: P.J.M. Maas, L. Cobb \& E.H.M. Grothe 4613

Herbarios: K, MO, U.

Nombre común: Desconocido.

Registro departamental: HU.

Regiones Ecológicas: MA; $2600 \mathrm{~m}$.

SINANPE: Sin registro.

Herbarios peruanos: Ninguno.

Observaciones: Este taxón fue descrito posterior a Brako \& Zarucchi (1993); no ha sido posible evaluarlo, ni asignarle una categoría.

\section{Myriocarpa laevigata Killip}

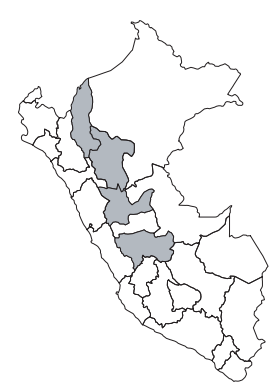

DD

Publicación: J. Wash. Acad. Sci. 15:55. 1925.

Colección tipo: J.F. Macbride 3925

Herbarios: F, US

Nombre común: Desconocido.

Registro departamental: AM, HU, JU, SM. Regiones Ecológicas: BMHM; 1830 $2100 \mathrm{~m}$.

SINANPE: Sin registro.

Herbarios peruanos: AMAZ (1), CPUN (1).

Observaciones: Liana conocida de matorrales en numerosas localidades del norte y centro del país, en las cuencas del Hualaga, Pozuzo, Mayo, Marañón. Se desconoce el estado de sus poblaciones.

\section{Phenax weddellianus Killip}

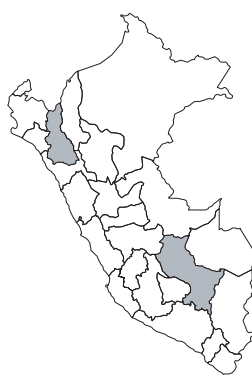

Publicación: J. Wash. Acad. Sci. 24(1): 43-44. 1934.

Colección tipo: C. Gay s.n.

Herbarios: P.

Nombre común: Desconocido.

Registro departamental: CA, CU.

Regiones Ecológicas: MA; $2300 \mathrm{~m}$.

SINANPE: Sin registro.

Herbarios peruanos: HUT (1).

Observaciones: Este taxón subarbustivo fue considerado por Brako \& Zarucchi (1993) como un endemismo; sin embargo, no ha sido posible evaluarlo, ni asignarle una categoría.

\section{Pilea citriodora Wedd.}

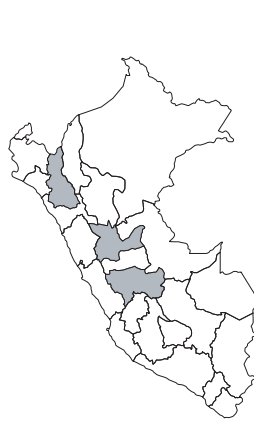

VU, B1a

Publicación: Ann. Sci. Nat., Bot., ser. 3, 18: 216. 1852.

Colección tipo: H. Ruiz \& J. Pavón s.n.

Herbarios: B.

Nombre común: Desconocido.

Registro departamental: CA, HU, JU.

Regiones Ecológicas: BMHM, BMHP; $800-2800 \mathrm{~m}$.

SINANPE: Sin registro.

Herbarios peruanos: AMAZ (1).

Observaciones: Arbusto conocido de varias localidades dispersas, en las cuencas del Palca, Huallaga y Marañón, donde ha sido recolectada en diferentes ocasiones, en matorrales o fragmentos boscosos intervenidos. 


\section{Pilea cushiensis Killip}

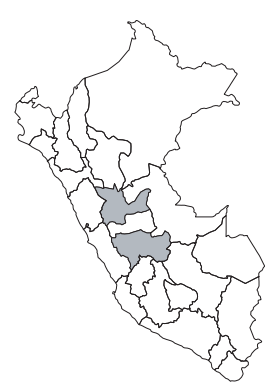

Publicación: Field Mus. Nat. Hist., Bot. Ser. 13(2/2): 340. 1937.

Colección tipo: J.F. Macbride

Macbride, J.F. 4826

Herbarios: F.

Nombre común: Desconocido.

Registro departamental: HU, JU.

Regiones Ecológicas: BMHM; 1500$2750 \mathrm{~m}$.

SINANPE: Sin registro.

Herbarios peruanos: Ninguno.

Observaciones: Este taxón fue considerado por Brako \& Zarucchi (1993) como un endemismo; sin embargo, no ha sido posible evaluarlo, ni asignarle una categoría.

\section{Pilea dombeyana Wedd.}

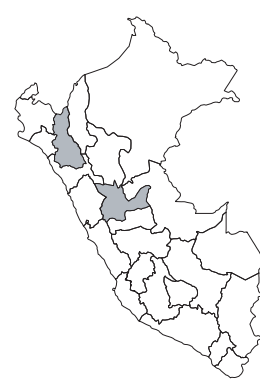

Publicación: Ann. Sci. Nat., Bot., ser. 3, 18: 221. 1852.

Colección tipo: J. Dombey s.n.

Herbarios: P.

Nombre común: Desconocido.

Registro departamental: CA, HU.

Regiones Ecológicas: MA, PSH; 2700$3500 \mathrm{~m}$.

SINANPE: Sin registro.

Herbarios peruanos: CPUN (2), HAO (1), USM (1).

Observaciones: Este taxón fue considerado por Brako \& Zarucchi (1993) como un endemismo; sin embargo, no ha sido posible evaluarlo, ni asignarle una categoría.

\section{Pilea foliosa Killip}

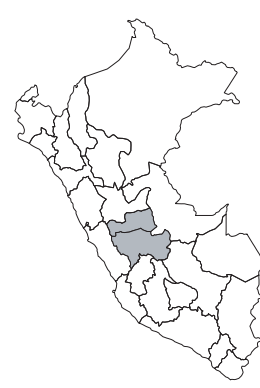

Publicación: Contr. U.S. Natl. Herb. 26(8):377. 1936

Colección tipo: E.P. Killip \& A.C. Smith 24400

Herbarios: MO, NY, US.

Nombre común: Desconocido.

Registro departamental: JU, PA.

Regiones Ecológicas: BPM, BMHM; $1900-3200 \mathrm{~m}$.

SINANPE: PNYC

Herbarios peruanos: Ninguno.

Observaciones: Este taxón fue considerado por Brako \& Zarucchi (1993) como un endemismo; sin embargo, no ha sido posible evaluarlo, ni asignarle una categoría.

\section{Pilea lamioides Wedd.}

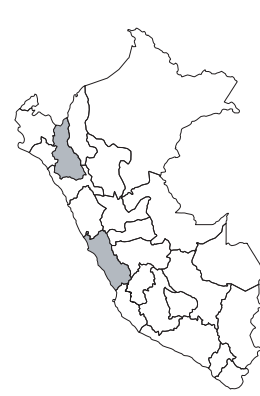

\section{VU, B1ab(iii)}

Publicación: Ann. Sci. Nat., Bot., ser. 3, 18: 213. 1852.

Colección tipo: J. Dombey s.n.

Herbarios: P.

Nombre común: Desconocido.

Registro departamental: CA, LI.

Regiones Ecológicas: DST, MA; 1400$2320 \mathrm{~m}$.

SINANPE: Sin registro.

Herbarios peruanos: CPUN (1).
Observaciones: Especie herbácea, descrita de una planta recolectada en el siglo XVIII, probablemente en la costa de Lima. Es conocida también de poblaciones aisladas en la vertiente occidental. Algunas de las localidades en Lima, como San Gerónimo (Amancaes) y Atocongo, mencionadas por Macbride (1936) están severamente deterioradas.

\section{Pilea macbridei Killip}

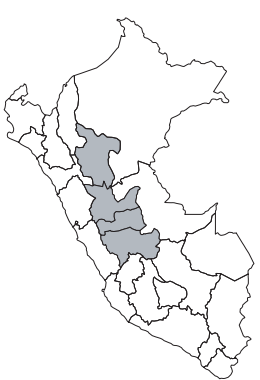

Publicación: J. Wash. Acad. Sci. 15: 52. 1925. Colección tipo: J.F. Macbride 5179

Herbarios: F.

Nombre común: Desconocido.

Registro departamental: HU, JU, PA, SM. Regiones Ecológicas: BPM; 2800-3000 m. SINANPE: Sin registro.

Herbarios peruanos: Ninguno.

Observaciones: Este taxón subarbustivo fue considerado por Brako \& Zarucchi (1993) como un endemismo; sin embargo, no ha sido posible evaluarlo, ni asignarle una categoría.

\section{Pilea macrocystolithica Killip}

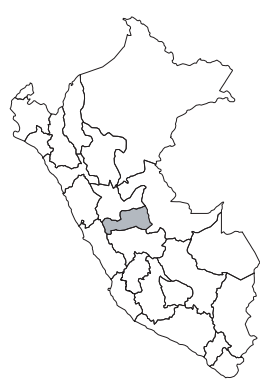

Publicación: Contr. U.S. Natl. Herb. 26(8): 384-385. 1936.

Colección tipo: T. Haenke 1860

Herbarios: PR.

Nombre común: Desconocido.

Registro departamental: PA.

Regiones Ecológicas: BMHM; $1850 \mathrm{~m}$.

SINANPE: PNYC

Herbarios peruanos: Ninguno.

Observaciones: Este taxón fue considerado por Brako \& Zarucchi (1993) como un endemismo; sin embargo, no ha sido posible evaluarlo, ni asignarle una categoría.

\section{Pilea nerteroides Killip}

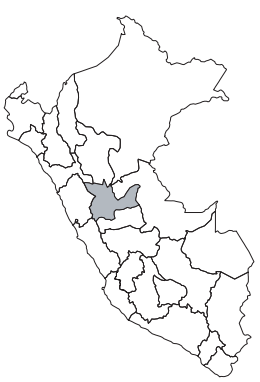

Publicación: Contr. U.S. Natl. Herb. 26(8): 377. 1936

Colección tipo: J.F. Macbride 4395

Herbarios: F, US.

Nombre común: Desconocido.

Registro departamental: HU.

Regiones Ecológicas: PSH; $4000 \mathrm{~m}$.

SINANPE: Sin registro.

Herbarios peruanos: Ninguno.

Observaciones: Este taxón fue considerado por Brako \& Zarucchi (1993) como un endemismo; sin embargo, no ha sido posible evaluarlo, ni asignarle una categoría.

\section{Pilea nummulariifolia (Sw.) Wedd. var. klugii Killip}

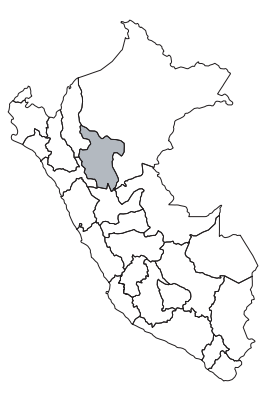

Publicación: Contr. U.S. Natl. Herb. 26: 513. 1939.

Colección tipo: G. Klug 4235

Herbarios: US.

Nombre común: Desconocido.

Registro departamental: SM.

Regiones Ecológicas: Sin datos; altitud desconocida.

SINANPE: Sin registro.

Herbarios peruanos: Ninguno. 
Observaciones: Este taxón fue considerado por Brako \& Zarucchi (1993) como un endemismo; sin embargo, no ha sido posible evaluarlo, ni asignarle una categoría.

\section{Pilea pavonii Wedd.}

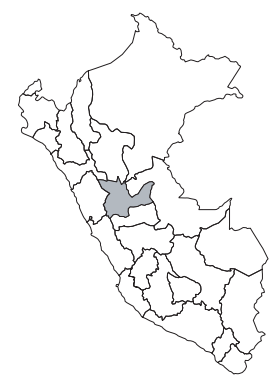

Publicación: Ann. Sci. Nat., Bot., ser. 3, 18: 219. 1852.

Colección tipo: H. Ruiz \& J. Pavón s.n.

Herbarios: B.

Nombre común: Desconocido.

Registro departamental: HU.

Regiones Ecológicas: BMHM; $2000 \mathrm{~m}$.

SINANPE: Sin registro.

Herbarios peruanos: Ninguno.

Observaciones: Este taxón fue considerado por Brako \& Zarucchi (1993) como un endemismo; sin embargo, no ha sido posible evaluarlo, ni asignarle una categoría.

\section{Pilea pichisana Killip}

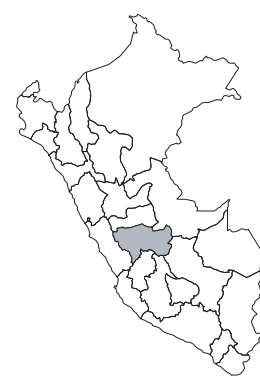

Publicación: Contr. U.S. Natl. Herb. 26(8): 383. 1936.

Colección tipo: E.P. Killip \& A.C. Smith 25876 Herbarios: US.

Nombre común: Desconocido.

Registro departamental: JU.

Regiones Ecológicas: BMHP; altitud desconocida.

SINANPE: Sin registro.

Herbarios peruanos: Ninguno.

Observaciones: Este taxón fue considerado por Brako \& Zarucchi (1993) como un endemismo; sin embargo, no ha sido posible evaluarlo, ni asignarle una categoría.

\section{Pilea pulegifolia (Poir.) Wedd.}

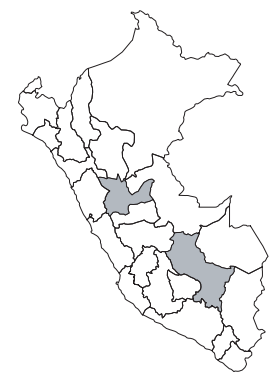

Publicación: Ann. Sci. Nat., Bot., ser. 3, 18: 218. 1852.

Colección tipo: J. Dombey s.n.

Herbarios: P.

Nombre común: Desconocido,

Registro departamental: CU, HU.

Regiones Ecológicas: Sin datos; altitud desconocida.

SINANPE: Sin registro.

Herbarios peruanos: Ninguno.

Observaciones: Este taxón fue considerado por Brako \& Zarucchi (1993) como un endemismo; sin embargo, no ha sido posible evaluarlo, ni asignarle una categoría.

\section{Pilea punctata (Kunth) Wedd.}

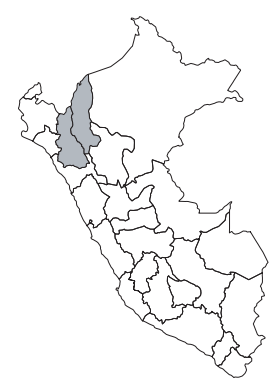

Publicación: Ann. Sci. Nat., Bot., ser. 3, 18: 222. 1852.

Colección tipo: A. Humboldt \& A. Bonpland s.n.

Herbarios: P.

Nombre común: Desconocido.

Registro departamental: AM, CA.

Regiones Ecológicas: MDE; $1600 \mathrm{~m}$.

SINANPE: Sin registro.

Herbarios peruanos: Ninguno.
Observaciones: Este taxón herbáceo fue considerado por Brako \& Zarucchi (1993) como un endemismo; sin embargo, no ha sido posible evaluarlo, ni asignarle una categoría.

\section{Pilea pusilla K. Krause}

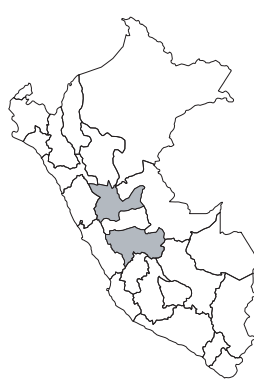

Publicación: Bot. Jahrb. Syst. 37: 530. 1906. Colección tipo: A. Weberbauer 2023

Herbarios: B.

Nombre común: Desconocido.

Registro departamental: HU, JU.

Regiones Ecológicas: BMHP; 1600$2000 \mathrm{~m}$.

SINANPE: Sin registro.

Herbarios peruanos: Ninguno.

Observaciones: Este taxón fue considerado por Brako \& Zarucchi (1993) como un endemismo; sin embargo, no ha sido posible evaluarlo, ni asignarle una categoría.

\section{Pilea ramosissima Killip}

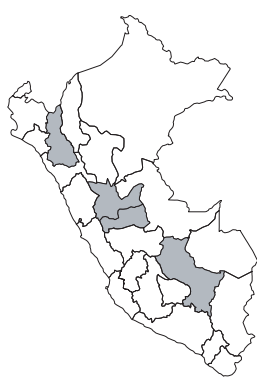

Publicación: Contr. U.S. Natl. Herb. 26(8): 380-381. 1936.

Colección tipo: J.F. Macbride 3650

Herbarios: F.

Nombre común: Desconocido.

Registro departamental: CA, CU, HU, PA.

Regiones Ecológicas: BPM; $2800 \mathrm{~m}$.

SINANPE: Sin registro.

Herbarios peruanos: Ninguno.

Observaciones: Este taxón subarbustivo fue considerado por Brako \& Zarucchi (1993) como un endemismo; sin embargo, no ha sido posible evaluarlo, ni asignarle una categoría.

\section{Pilea subamplexicaulis Killip}

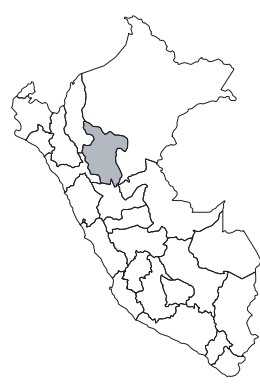

Publicación: Contr. U.S. Natl. Herb. 26(8): 390. 1936.

Colección tipo: E.H.G. Ule 6588

Herbarios: US.

Nombre común: Desconocido.

Registro departamental: SM.

Regiones Ecológicas: BMHP; 1100$1500 \mathrm{~m}$.

SINANPE: Sin registro.

Herbarios peruanos: Ninguno.

Observaciones: Este taxón herbáceo fue considerado por Brako \& Zarucchi (1993) como un endemismo; sin embargo, no ha sido posible evaluarlo, ni asignarle una categoría.

\section{Pilea suffruticosa Killip}

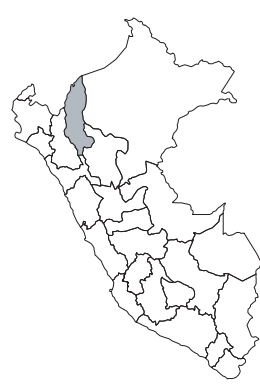

Publicación: Bot. Jahrb. Syst. 37: 529. 1906. Colección tipo: A. Weberbauer 4387 Herbarios: US.

Nombre común: Desconocido. Registro departamental: AM. Regiones Ecológicas: BMHM; $2500 \mathrm{~m}$. SINANPE: Sin registro.

Herbarios peruanos: Ninguno. 
Observaciones: Este taxón subarbustivo fue considerado por Brako \& Zarucchi (1993) como un endemismo; sin embargo, no ha sido posible evaluarlo, ni asignarle una categoría.

\section{Pilea verrucosa Killip}

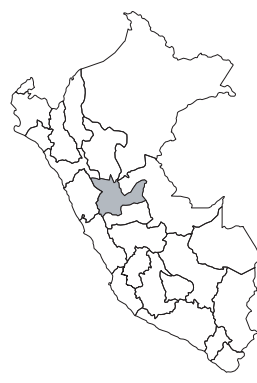

Publicación: J. Wash. Acad. Sci. 15: 53. 1925.

Colección tipo: J.F. Macbride 5201

Herbarios: F, US.

Nombre común: Desconocido.

Registro departamental: HU,

Regiones Ecológicas: BPM; $2800 \mathrm{~m}$.

SINANPE: Sin registro.

Herbarios peruanos: Ninguno.

Observaciones: Este taxón arbustivo fue considerado por Brako \& Zarucchi (1993) como un endemismo; sin embargo, no ha sido posible evaluarlo, ni asignarle una categoría.

\section{Pilea weberbaueri Killip}

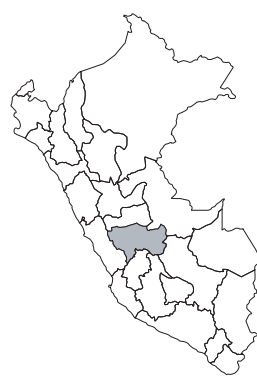

Publicación: Contr. U.S. Natl. Herb. 26(8): 380. 1936.

Colección tipo: A. Weberbauer 2022

Herbarios: US; MOL!

Nombre común: Desconocido.

Registro departamental: JU.

Regiones Ecológicas: BMHP; $1900-$ $2000 \mathrm{~m}$.

SINANPE: Sin registro

Herbarios peruanos: MOL (isotipo).

Observaciones: Este taxón fue considerado por Brako \& Zarucchi (1993) como un endemismo; sin embargo, no ha sido posible evaluarlo, ni asignarle una categoría.

\section{Pouzolzia longipes Killip}

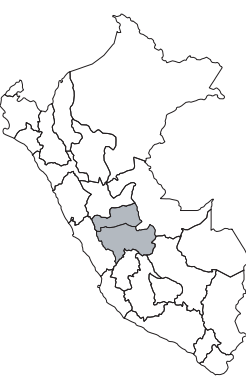

Publicación: Field Mus. Nat. Hist., Bot. Ser. 13(2/2): 364. 1937

Colección tipo: E.P. Killip \& A.C. Smith 25753

Herbarios: F, NY.

Nombre común: Desconocido.

Registro departamental: JU, PA.

Regiones Ecológicas: BMHM; 1600$1900 \mathrm{~m}$.

SINANPE: Sin registro,

Herbarios peruanos: Ninguno.

Observaciones: Hierba monoica, considerada por Brako \& Zarucchi (1993) como un endemismo; sin embargo, no ha sido posible evaluarlo, ni asignarle una categoría.

\section{Urtica lalibertadensis Weigend}

DD

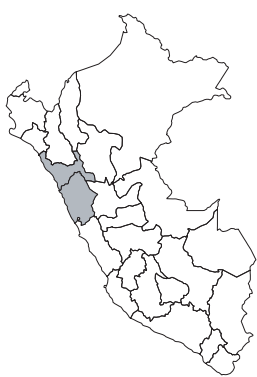

Publicación: Rev. Per. Biol. 12(2): 259260, f. 5a-c. 2005.

Colección tipo: M. Weigend \& Schwarzer 8018

Herbarios: B; HUT, USM!.

Nombre común: Desconocido.

Registro departamental: AN, LL.

Regiones Ecológicas: MA; 3043-3550

m.

SINANPE: Sin registro.

Herbarios peruanos: HUT (isotipo), USM (holotipo).

Observaciones: Especie herbácea escandente, conocida de varias localidades, en la cuenca del Marañón y del Santa. Aparentemente está relacionada con Urtica leptostachya (Weigend \& Rodriguez, 2005). Fue descrita de una planta recolectada en 2004. Se desconoce el estado de sus poblaciones.

\section{Urtica peruviana Geltman}

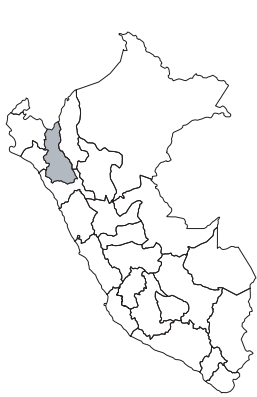

DD

Publicación: Novon 8(1): 15-16, f. 1. 1998.

Colección tipo: A. Sagástegui A. et al. 12630

Herbarios: MO; $\mathrm{HAO!}$.

Nombre común: Ishguin.

Registro departamental: CA.

Regiones Ecológicas: MA; 2400—2800

$\mathrm{m}$.

SINANPE: Sin registro.

Herbarios peruanos: HAO (isotipo+2), HUT (isotipo+4?).

Observaciones: Especie herbácea conocida de la vertiente del Pacífico en el sur-occidente de Cajamarca, entre las cuencas del Magdalena y Chicama. Se desconoce el estado de sus poblaciones.

\section{Urtica urentivelutina Weigend}

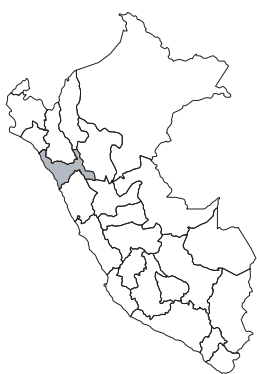

DD

Publicación: Rev. Per. Biol. 12(2): 253254, f. 2-3b 2005.

Colección tipo: M. Weigend \& Schwarzer 7907

Herbarios: B; HUT, USM!.

Nombre común: Desconocido.

Registro departamental: LL.

Regiones Ecológicas: MA; 3048 m.

SINANPE: Sin registro.

Herbarios peruanos: USM (holotipo).

Observaciones: Planta dioica, conocida y descrita de plantas recolectada en el 2004, de los alrededores de Molino Viejo, en la cuenca del Marañón. El ejemplar tipo representa a la planta masculina. Esta especie aparentemente está relacionada a Urtica macbridei de Perú y Ecuador. Se desconoce el estado de sus poblaciones. 